\title{
1 Pulse Shape Discrimination Performance of Stilbene Coupled 2 to Low-Noise Silicon Photomultipliers
}

4 Abstract

5 Pulse shape discrimination (PSD) techniques can be used to discern between neutron and 6 gamma-ray interactions in certain organic scintillators. Traditionally, photomultiplier tubes 7 (PMTs) have been used in organic-scintillator assemblies. However, silicon photomultipliers 8 (SiPMs) have great potential to be used in many applications in which PMTs have been 9 predominantly used, including those utilizing PSD techniques. To evaluate the current state of

10 the art of the SiPM technology, SensL's 6-mm B-Series and C-Series SiPMs were compared to a

11 fast Hamamatsu PMT in conjunction with a $6 \times 6 \times 6-\mathrm{mm}^{3}$ stilbene organic scintillator to assess the

12 PSD performance of the detector assemblies. Measurements with a Cf-252 source were

13 performed and a figure of merit (FOM) for discriminating between neutron and gamma-ray 14 pulses between $100 \mathrm{keVee}$ and $200 \mathrm{keVee}$ was calculated for each assembly. A digital charge-

15 integration PSD technique was used to process all measured data. The FOM for the B-Series 16 SiPM, PMT, and C-Series SiPM was 1.37, 1.93, and 2.13, respectively. The C-Series SiPM was 17 shown to perform as well as the PMT in the experiments.

\section{Keywords}

Silicon photomultiplier, pulse shape discrimination, stilbene, neutron detection, organic 25 scintillator

\section{Introduction}

Silicon photomultipliers (SiPMs) are solid-state light sensors with similar lightconversion and amplification capabilities to photomultiplier tubes (PMTs) [1,2]. When coupled to a scintillating medium, these devices have been used in a variety of radiation detection and spectroscopy applications including dosimetry [3, 4], medical imaging [5-8], high-energy physics [9-11], and homeland security [12-14]. Additionally, organic crystals with pulse shape discrimination (PSD) capabilities coupled to SiPMs have been shown to be capable of fast- 
neutron detection [15-17]. Previous approaches used complex PSD algorithms and exhibited poorer PSD performance with SiPMs when compared to PMTs. Recently, however, improvements in SiPM technology have significantly reduced the amount of noise produced [18]. This noise reduction opens up the potential for improved PSD performance of SiPMs coupled to organic scintillators. SiPMs already have a number of attractive features when compared to PMTs, including low sensitivity to magnetic fields, low voltage requirements, small size, and competitive cost. If the PSD performance of SiPMs matched that of PMTs, SiPMs could present a viable alternative to PMTs in applications where PSD performance is critical. PSD-capable organic scintillators are used in a variety of applications, including nonproliferation and safeguards applications [19-21], fusion experiments [22], and geological studies [23]. This work investigates the PSD performance of stilbene read-out with the new generation of SiPMs and compares it to the performance of the previous SiPM generation and a fast PMT.

\section{Silicon Photomultipliers}

SiPMs are composed of numerous Geiger-mode avalanche photodiodes (APDs), referred to as microcells [1]. In particular, the SiPMs used in this work are each composed of 18980 35$\mu \mathrm{m}$ microcells $[24,25]$. The outputs of the microcells are summed up, producing a signal proportional to the number of microcells discharging at any time [1]. While a single APD gives a binary response indicating whether or not a photon was detected, SiPMs are capable of producing a signal proportional to the incident photon flux. Furthermore, when sensing scintillation light of low enough intensity such that all of the microcells do not trigger at once, a time profile of the light production is retained in the output signal of the SiPM. The preservation of the temporal distribution of scintillation light production, originating in a PSD-capable scintillator, allows PSD to be performed.

The SiPMs used in this work have peak sensitivity at approximately $420 \mathrm{~nm}[24,25]$. In comparison, stilbene's wavelength of maximum emission is approximately $410 \mathrm{~nm}$ [26]. This peak-sensitivity similarity makes these SiPMs a good option for measuring scintillation from stilbene.

\section{Method}

Three light sensors were evaluated: a Hamamatsu H10580 PMT assembly (R9800 PMT), a SensL MicroFB-60035 SiPM, and a SensL MicroFC-60035 SiPM. A single 6x6x6-mm ${ }^{3}$ stilbene crystal from Proteus, Inc. [27] was coupled to each of the light sensors. Eljen Technology EJ-550 silicon optical grease was used to couple the stilbene crystal to each of the light sensors. The stilbene crystal coupled to the PMT and the B-Series SiPM is shown in Fig. 1 and Fig. 2, respectively. During experiments, each detector assembly was contained within a custom-designed, opaque, 3D-printed coupler, which prevented external light from reaching the light-sensitive portions of the assemblies. A CAEN V1730 digitizer was used to digitize and 
collect the pulses from the PMT and B-Series configurations and a CAEN DT5730 was used to digitize pulses from the C-Series configuration. Both digitizers have 14-bit resolution and a sampling frequency of $500 \mathrm{MHz}$ [28]. The different digitizers were used because of limited availability and comparison measurements were performed to confirm that their performance is similar enough to justify their use. The resulting figures of merit, described in section 4.2 , agreed within approximately $10 \%$.

75

76

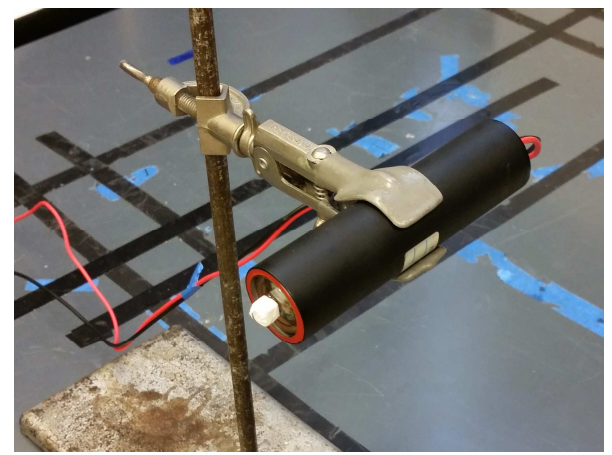

Fig. 1. Photograph of a stilbene crystal coupled to a Hamamatsu H10580 PMT assembly.

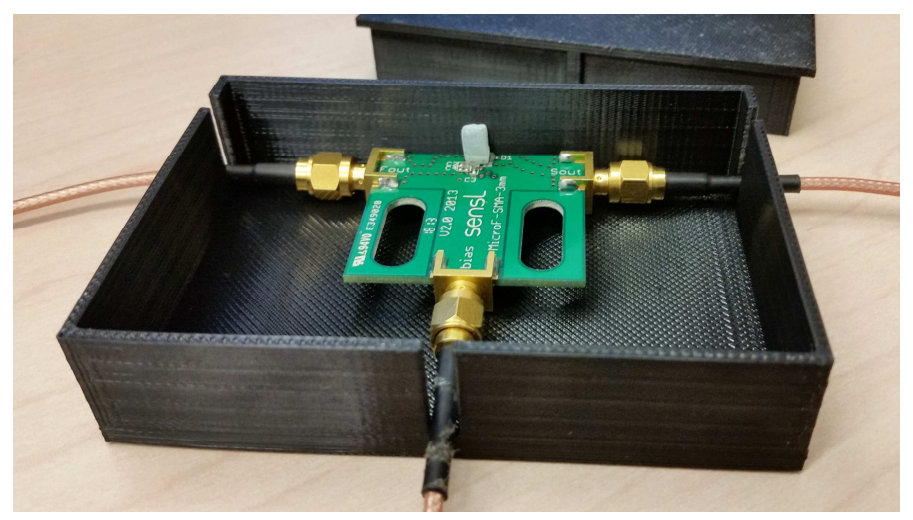

Fig. 2. Photograph of a stilbene crystal coupled to a SensL MicroFB-60035 (B-series) SiPM.

Each detector configuration was calibrated using 478-keVee Compton edge measured with 662$\mathrm{keV}$ gamma rays from a Cs-137 source. Then, each configuration was used to acquire pulses while placed $5 \mathrm{~cm}$ from a bare Cf-252 spontaneous fission source, with a source strength of approximately 150,000 neutrons per second. A 50-keVee threshold was used for each measurement.

PSD was performed using a digital charge comparison technique in which the ratio of the integral of the pulse tail to the total integral of each pulse is calculated to identify the type of incident radiation [29, 30]. Three parameters were varied for each detector configuration in order to obtain the optimum particle discrimination: the time before the pulse peak at which the total integral begins, the time after the pulse peak at which the tail integral begins, and the time after the pulse peak at which both integrals end. To optimize these values, each combination of plausible values was used to calculate a figure of merit (FOM) using an automated MATLAB script. The FOM quantifies the quality of particle discrimination, as discussed in section 4.2. The 
92 combination for each detector configuration that produced the largest figure of merit was used.

93 These optimal values are listed in Table 1 for all three configurations. It is worth noting that for

94 the C-Series SiPM, the integral end time is significantly longer than that of the B-Series SiPM.

95 This is because of the lower noise in the tail of the pulses, as discussed in section 4.1.

Table 1. Optimized PSD Parameters.

\begin{tabular}{lccc}
\hline Configuration & $\begin{array}{c}\text { Total Start } \\
\text { Time (ns) }\end{array}$ & $\begin{array}{c}\text { Tail Start } \\
\text { Time (ns) }\end{array}$ & $\begin{array}{c}\text { Integral End } \\
\text { Time (ns) }\end{array}$ \\
\hline PMT & 2 & 18 & 200 \\
B-Series SiPM & 2 & 34 & 520 \\
C-Series SiPM & 0 & 44 & 1100 \\
\hline
\end{tabular}

4. Results

\subsection{Waveforms}

A digitized neutron and a gamma-ray pulse acquired using the PMT configuration is shown in Fig. 3. While the two example waveforms have approximately the same pulse height, the neutron pulse has a larger tail than the gamma-ray pulse. This expected phenomenon is exploited to perform PSD.

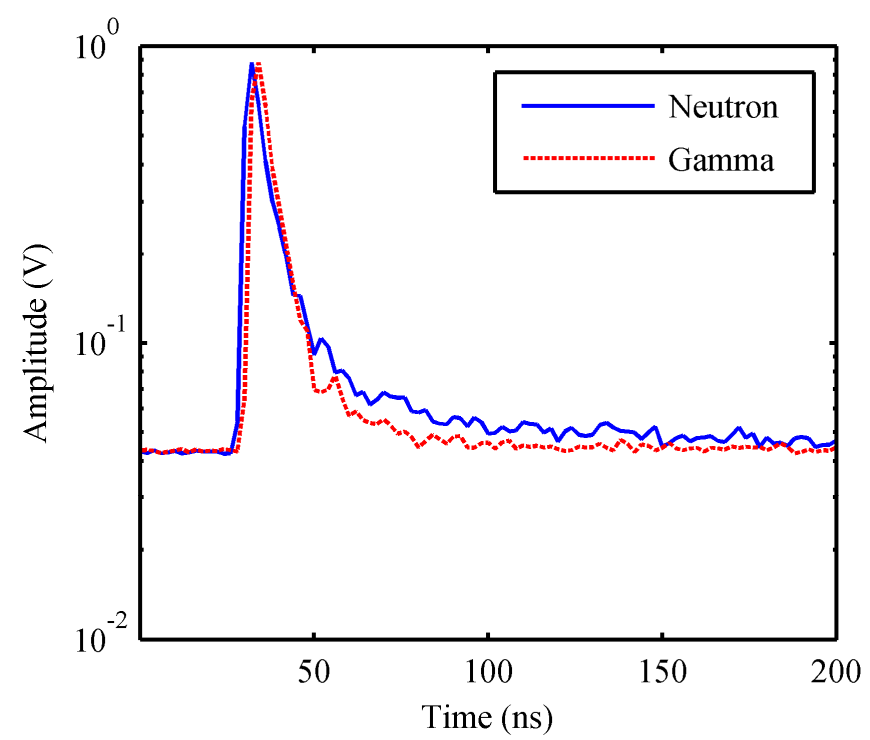

Fig. 3. Digitized 1-MeVee neutron and gamma-ray pulses measured using stilbene coupled to a PMT.

105 Because of the fast timing properties of the PMT, the digitized signal closely represents the timescale in which the light decay occurs within the stilbene crystal. This is in contrast to the longer response of the B-Series SiPM, which, like the C-Series SiPM, has a microcell recovery time of 210 ns [24, 25], as exemplified by the pulses shown in Fig. 4. Nonetheless, the tail of the neutron pulse acquired with this device is still substantially larger than that of the corresponding gamma ray, allowing PSD to be performed to distinguish between the two particle types. 


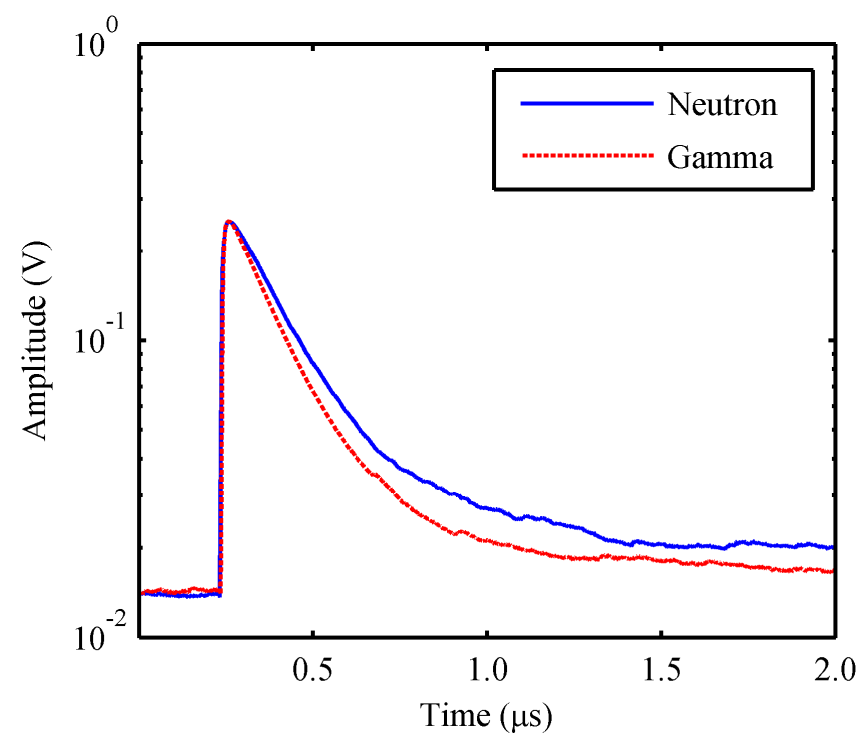

Fig. 4. Digitized 1-MeVee neutron and gamma-ray pulses measured using stilbene coupled to a B-Series SiPM.

113 Fig. 5 shows pulses acquired using the C-Series SiPM; these appear similar in shape to the B-

114 Series SiPM pulses but exhibit less noise in their baseline, allowing for more accurate

115 discrimination between particle types. The amount of noise in the pulse is quantified by

116 measuring the root mean square (RMS) noise in the baseline of the pulses. A histogram of the

117 RMS noise for 40 2-ns samples in the baseline for each of the detector configurations is plotted

118 in Fig. 6. The C-Series SiPM pulses have an average RMS noise of $0.108 \mathrm{mV}$ whereas it is 0.137

$119 \mathrm{mV}$ for the B-Series SiPM pulses.

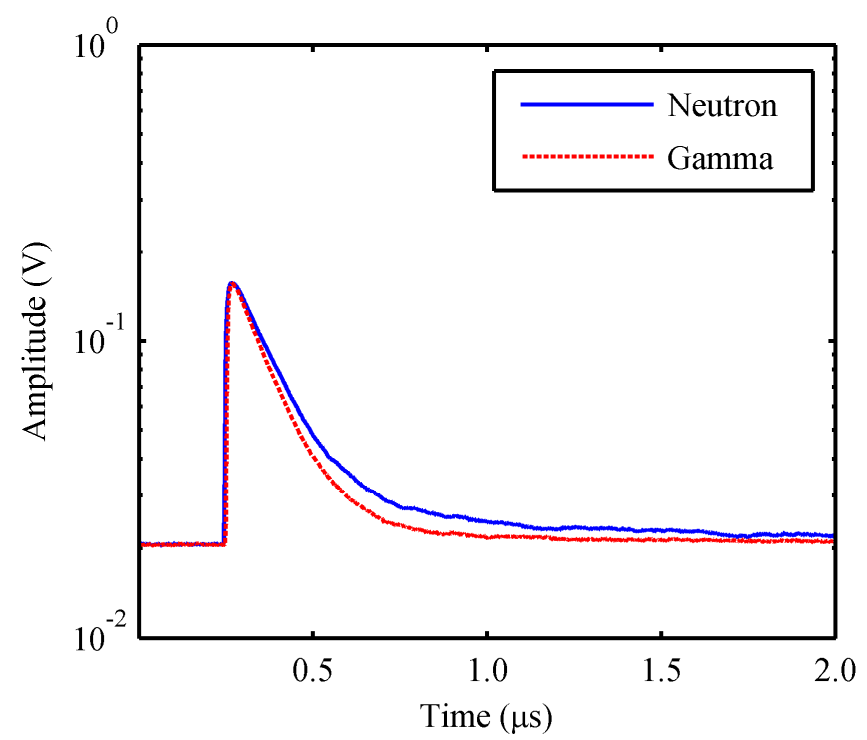




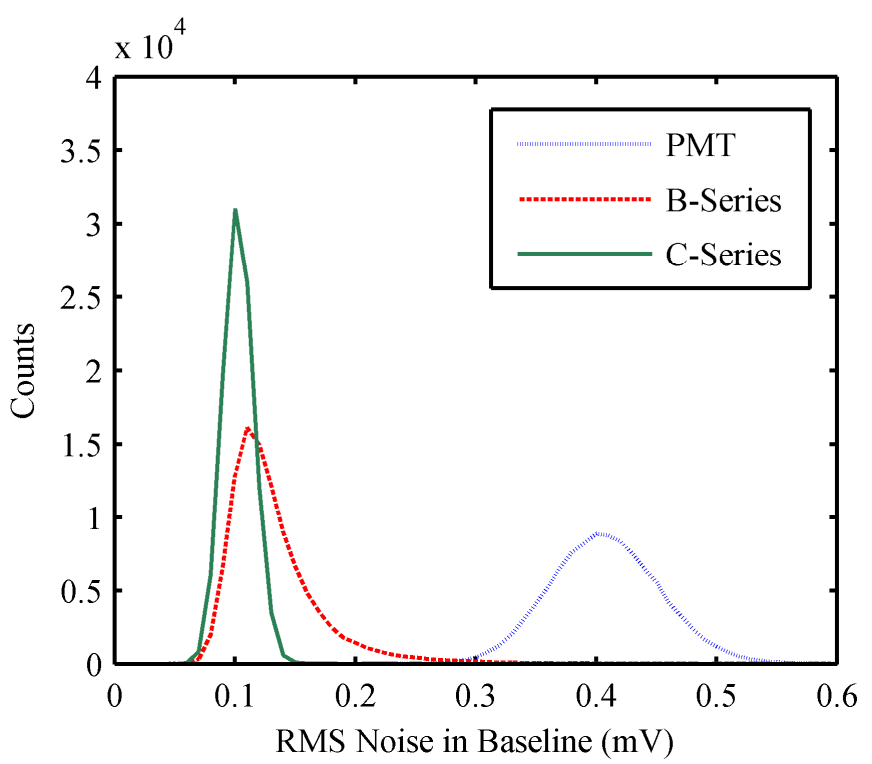

Fig. 6. Histogram of root mean square noise in 40 2-ns samples for 100,000 waveforms.

\subsection{PSD}

Plots of tail to total ratio versus pulse height for the PMT configuration, B-Series SiPM configuration, and C-series SiPM configuration are shown in Fig. 7, Fig. 8, and Fig 9, respectively. The threshold of the presented data is $50 \mathrm{keVee}$. Tail to total ratios were calculated using the technique described in section 3 . In each case, two regions can be clearly seen: a higher tail to total ratio region which corresponds to neutrons, and a lower tail to total ratio region resulting from gamma-ray interactions. In all cases, there is good separation between the two regions for large pulse heights, corresponding to high-energy depositions. However, there is some overlap between the regions for low-energy depositions [31]. There is significantly more overlapping of the regions for the B-Series SiPM configuration as compared to the case of the PMT configuration. However, even at low energy, the C-Series SiPM configuration shows as good separation between the two regions as the PMT case.

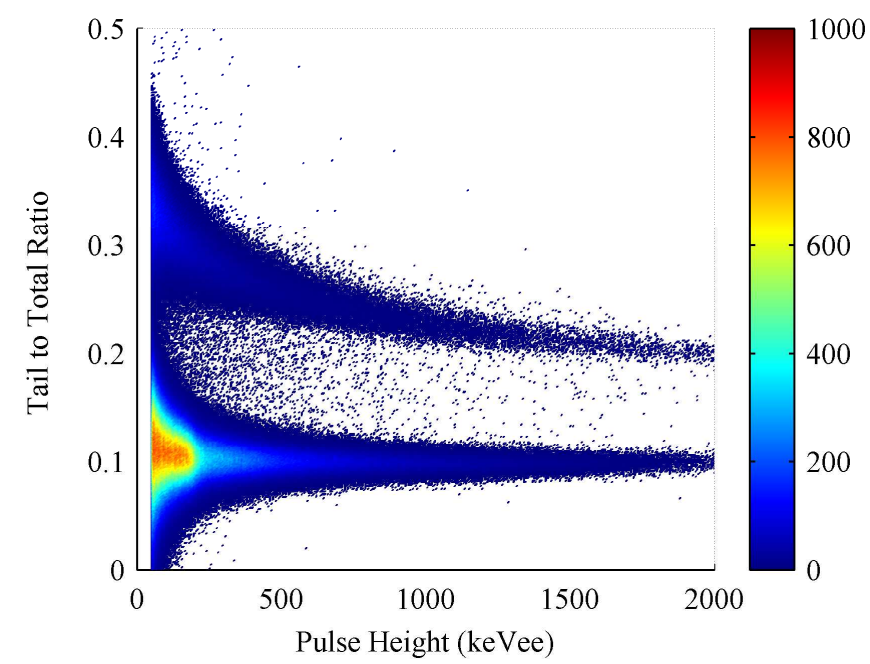




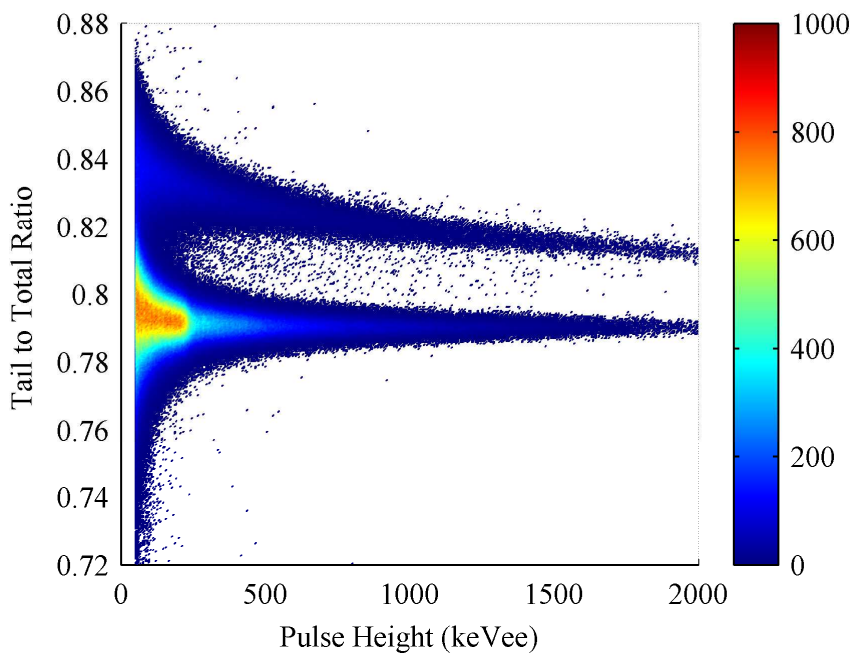

Fig. 8. Tail to total ratio versus pulse height for 2,000,000 pulses produced by a stilbene crystal coupled to a B-Series SiPM measuring Cf-252.

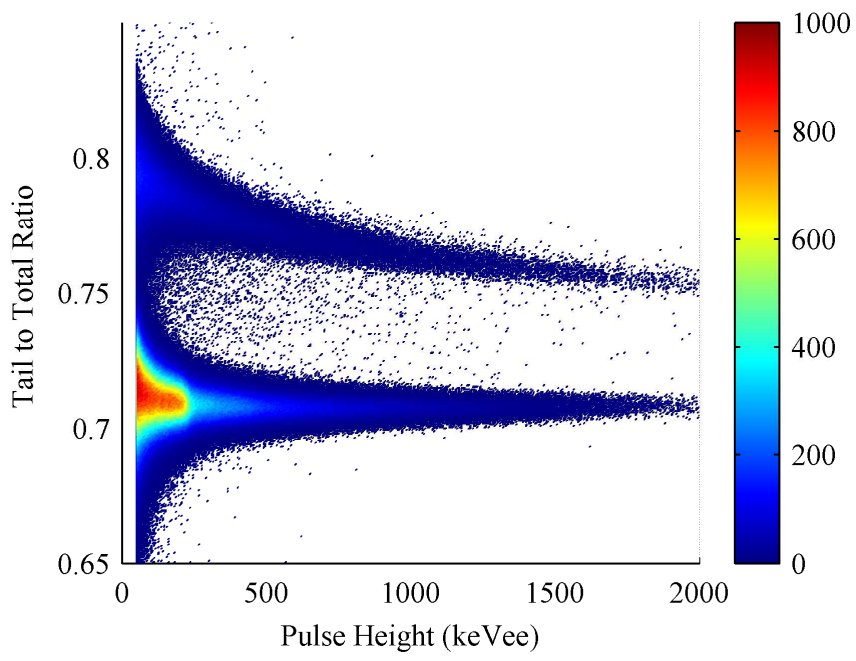

Fig. 9. Tail to total ratio versus pulse height for 2,000,000 pulses produced by a stilbene crystal coupled to a C-Series SiPM measuring Cf-252.

A histogram of the tail to total ratio of pulses corresponding to light outputs between 100 keVee and $200 \mathrm{keVee}$ for each detector configuration is shown in Fig. 10. This range corresponds to approximately $1 \mathrm{MeV}$ of energy deposited by a neutron in the stilbene scintillator. Two peaks are seen for each detector configuration. The peak centered on the lower tail to total ratio corresponds to gamma-ray pulses and the peak at the higher tail to total ratio results from neutron pulses. Again, there is some overlap between the two peaks for the B-Series SiPM configuration but excellent separation is seen for both the PMT configuration and C-Series SiPM detector. The separation between the gamma-ray and neutron regions can be quantified using a figure of merit. The figure of merit used in this work is calculated as

$$
F O M=\frac{S}{F W H M_{n}+F W H M_{\gamma}}
$$


152 where $S$ is the separation between the neutron and gamma-ray peaks, respectively, and $F W H M_{n}$ 153 and $F W H M_{\gamma}$ are the full width at half max of the corresponding peaks [32, 33]. The FOM for the 154 PMT configuration, B-Series SiPM configuration, and C-Series configuration is 1.93, 1.37, and 155 2.13, respectively. While the FOM for the B-Series SiPM configuration is lower than that of the 156 PMT-based assembly, the FOM of the C-Series configuration exceeds that of both of the other 157 two configurations. Additionally, the C-Series SiPM data was processed using the PSD 158 parameters listed in Table 1 that were optimized for the B-Series SiPM. The resultant FOM, 159 1.93, is still substantially better than the B-Series SiPM results but better PSD is achieved with 160 the parameters optimized for the C-Series SiPM.

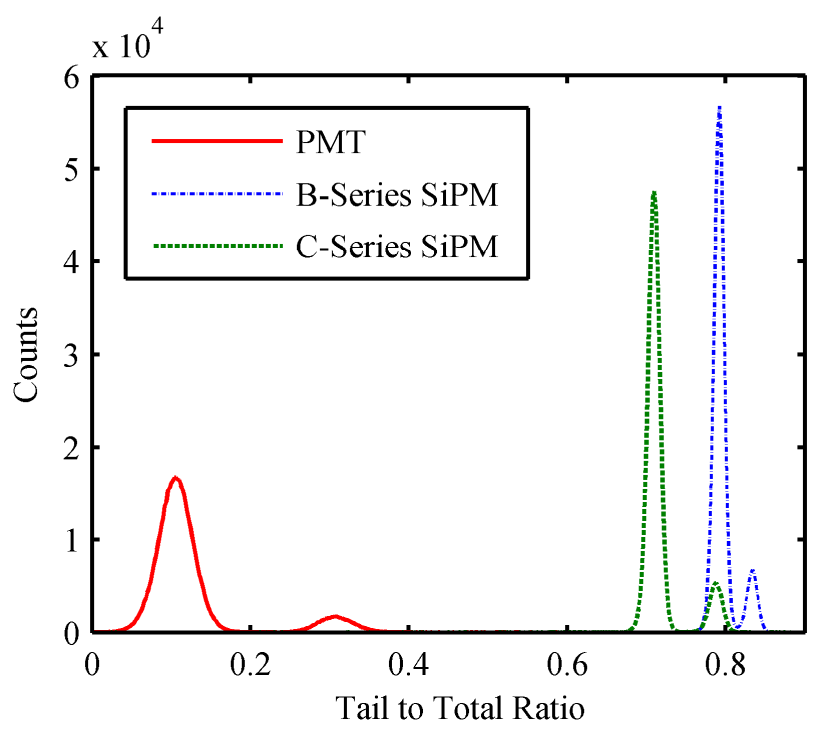

Fig. 10. Histogram of the tail to total integral ratio for 500,000 pulses between 100 keVee and 200 keVee from a measurement of Cf-252 using a stilbene crystal coupled to a SiPM and a PMT.

As mentioned in section 3, two different digitizers were used in order to perform the aforementioned measurements. Although the two digitizers used (CAEN V1730 and CAEN DT5730) have identical specifications [28], an experiment was performed in order to verify a valid comparison of FOM between measurements using the different digitizers. The C-Series SiPM was coupled to a $6 \times 6 \times 6-\mathrm{mm}^{3}$ stilbene crystal from Inrad Optics [34]. This detector configuration was placed $5 \mathrm{~cm}$ from the same $\mathrm{Cf}-252$ used in the experimental setup described in section 3. Each digitizer was used to acquire pulses from the new experimental setup and the analysis described previously in this section was repeated. The FOMs determined from the pulses acquired using the V1730 and DT5730 were 2.06 and 2.29, respectively. The $10 \%$ difference in FOM using the two different digitizers is similar to the $9 \%$ difference in FOM between the PMT configuration and the C-Series configuration. Therefore, although the FOM determined for the $\mathrm{C}$-Series configuration is larger than that for the PMT configuration, the PSD performance of the two should be considered as approximately equal.

\subsection{Pulse Height Distributions}

Separate neutron and gamma-ray pulse height distributions (PHDs) for each detector configuration are shown in Fig. 11, with features that are consistent with a measurement of an aged Cf-252 fission source. For example, a feature can be seen in the gamma-ray PHDs near 234 
181 keVee, corresponding to the Compton edges of the 388- and 333-keV gamma rays emitted from 182 the Cf-249 present in the Cf-252 fission source. Because of the excellent PSD at that energy for 183 all three configurations, there is no corresponding feature in the neutron PHDs. The source to 184 detector distance was not held exactly constant between these measurements, but this is not 185 important for the sake of evaluating PSD performance. Fig. 11 is shown to illustrate similarities 186 among the measured PHDs; however, the integrals of the PHDs should not be compared directly.

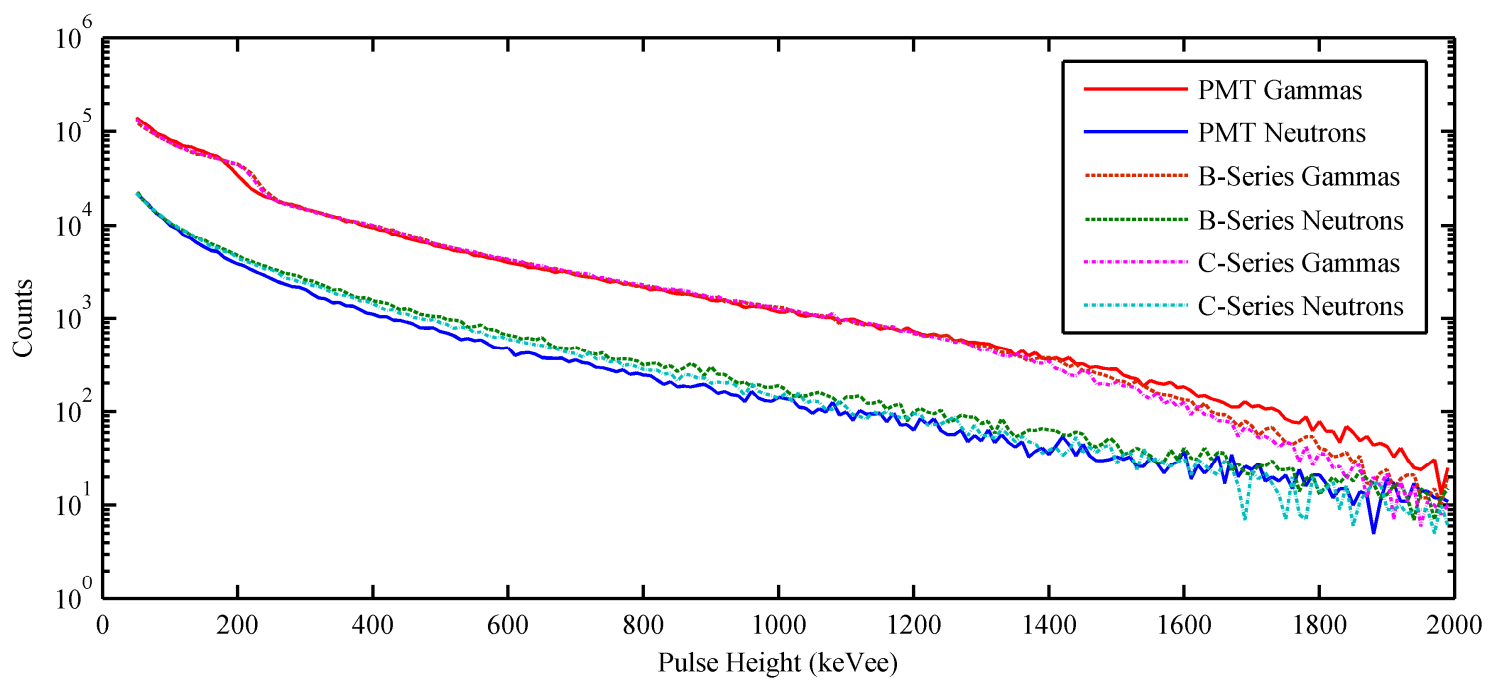

Fig. 11. Separate neutron and gamma-ray pulse height distributions for a measurement of Cf-252 using stilbene coupled PMT, B-Series SiPM, and C-Series SiPM.

\section{Conclusion}

The study verifies the reduction in noise of the latest generation of SiPMs and shows the consequent improvement in PSD performance of SiPMs when coupled to PSD-capable organic scintillators. The FOMs for the B-Series SiPM, PMT, and C-Series SiPM configurations were 1.37, 1.93, and 2.13, respectively, suggesting that SiPMs can now perform as well as PMTs for PSD applications. At the same time, SiPMs have some potential drawbacks, including the production of long pulses and nonlinearity at high energies, which should be considered when selecting a photomultiplier technology for a specific application. Nonetheless, the recent improvement in SiPM technology provides an exciting opportunity for their use to be explored for a variety of fast-neutron/gamma-ray detection applications.

\section{Acknowledgment}

We would like to thank John Murphy from SensL for lending us the SiPMs used in these studies. We would also like to thank Mark Norsworthy for proof reading this manuscript. This research was performed under appointment to the Nuclear Nonproliferation International Safeguards Graduate Fellowship Program sponsored by the National Nuclear Security Administration's Next Generation Safeguards Initiative (NGSI). In addition, this work was 
206

207

208

209

210

211

212

213

214

215

216

217

218

219

220

221

222

223

224

225

226

227

228

229

230

231

232

233

234

235

236

237

238

239

240

funded in-part by the Consortium for Verification Technology under Department of Energy

National Nuclear Security Administration award number DE-NA0002534.

\section{References}

1. D.J. Herbert, V. Saveliev, N. Belcari, N. D'Ascenzo, A. Del Guerra, A. Golovin, First results of scintillator readout with silicon photomultiplier, IEEE Transactions on Nuclear Science 53 (1) (2006) 389-394.

2. J. Ninković, Recent developments in silicon photomultipliers, Nuclear Instruments and Methods in Physics Research Section A: Accelerators, Spectrometers, Detectors and Associated Equipment 580 (2) (2007) 1020-1022.

3. L.M. Moutinho, I.F. Castro, L. Peralta, M.C. Abreu, J.F.C.A. Veloso, Development of a scintillating optical fiber dosimeter with silicon photomultipliers, Nuclear Instruments and Methods in Physics Research Section A: Accelerators, Spectrometers, Detectors and Associated Equipment 735 (2014) 640-643.

4. S.F. Jackson, S.D. Monk, S.J. Stanley, K.Lennox, Sub-aquatic response of a scintillator, fibre optic and silicon photomultiplier based radiation sensor, Nuclear Instruments and Methods in Physics Research Section A: Accelerators, Spectrometers, Detectors and Associated Equipment 752 (2014) 42-46.

5. R.R. Raylman, A. Stolin, S. Majewski, J. Proffitt, A large area, silicon photomultiplierbased PET detector module, Nuclear Instruments and Methods in Physics Research Section A: Accelerators, Spectrometers, Detectors and Associated Equipment 735 (2014) 602-609.

6. G. Llosa, N. Belcari, M.G. Bisogni, G. Collazuol, S. Marcatili, P. Barrillon, C. de La Taille, S. Bondil-Blin, N. Dinu, M. Melchiorri, A. Tarolli, C. Piemonte, A. Del Guerra, Energy, Timing and position resolution studies with 16-pixel silicon photomultiplier matrices for small animal PET, IEEE Transactions on Nuclear Science 56 (5) (2009) 2586-2593.

7. N. D'Ascenzo, V. Saveliev, Study of silicon photomultipliers for the medical imaging systems, Nuclear Instruments and Methods in Physics Research Section A: Accelerators, Spectrometers, Detectors and Associated Equipment 695 (2012) 265-267.

8. A.V. Stolin, S. Majewski, G. Jaliparthi, R.R. Raylman, Construction and evaluation of a prototype high resolution, silicon photomultiplier-based, tandem positron emission tomography system, IEEE Transactions on Nuclear Science 60 (1) (2013) 82-86.

9. A. Sótér, K. Todoroki, T. Kobayashi, D. Barna, D. Horváth, M. Hori, Segmented scintillation detectors with silicon photomultiplier readout for measuring antiproton annihilations, Review of Scientific Instruments (85) (2014). 
10. P.F. Bloser, J.S. Legere, C.M. Bancroft, L.F. Jablonski, J.R. Wurtz, C.D. Ertley, M.L. McConnell, J.M. Ryan, Testing and simulation of silicon photomultiplier readouts for scintillators in high-energy astronomy and solar physics, Nuclear Instruments and Methods in Physics Research Section A: Accelerators, Spectrometers, Detectors and Associated Equipment 763 (2014) 26-35. Corso, F. Evangelisti, C. Fanin, E. Feltresi, N. Gagliardi, E. Luppi, R. Malaguti, M. Manzali, M. Melchiorri, M. Munerato, M. Posocco, A.C. Ramusino, M. Rotondo, R. Stroili, L. Tomassetti, A scintillator based muon system with SiPM readout for the SuperB detector,in: Proceedings of the IEEE Nuclear Science Symposium and Medical Imaging Conference, Knoxville, TN, October 30-November 6, 2010.

12. L. Sinclair, P. Saull, D. Hanna, H. Seywerd, A. Macleod, P. Boyle, Silicon photomultiplier-based compton telescope for safety and security (SCoTSS), IEEE Transactions on Nuclear Science 61 (5) (2014) 2745-2752.

13. H.M. Park, S.J. Jeon, H.K. Lee, K.S. Joo, Design of a silicon photomultiplier based compact radiation detector for Homeland Security screening, in: Proceeding of the Advancements in Nuclear Instrumentation Measurement Methods and their Applications Conference, Marseille, France, June 23-27, 2013.

14. R. Bencardino, J.E. Eberhardt, Development of a fast-neutron detector with silicon photomultiplier readout, IEEE Transactions on Nuclear Science 56 (3) (2009) 1129-1134.

15. M.L. Ruch, C.B. Sivels, S.A. Czyz, M. Flaska, S.A. Pozzi, Comparison between silicon photomultipliers and photomultiplier tubes for pulse shape discrimination with stilbene, in: Proceedings of the IEEE Nuclear Science Symposium and Medical Imaging Conference, Seattle, WA, November 8-15, 2014.

16. R.M. Preston, J.E. Eberhardt, J.R. Tickner, Neutron-gamma pulse shape discrimination using organic scintillators with silicon photomultiplier readout, IEEE Transactions on Nuclear Science 61 (4) (2014) 2410-2418.

17. M. Grodzicka, T. Szczęśniak, M. Moszyński, D. Wolski, L. Swiderski, K. Grodzicki, S. Korolczuk, J. Baszak, P. Schotanus, Study of n- $\gamma$ discrimination by zero-crossing method with SiPM based scintillation detectors, in Proceedings of the IEEE Nuclear Science Symposium and Medical Imaging Conference, Seattle, WA, November 8 - 15, 2014.

18. K. O'Neill, C. Jackson, SensL B-Series and C-Series silicon photomultipliers for time-offlight positron emission tomography, Nuclear Instruments and Methods in Physics Research Section A: Accelerators, Spectrometers, Detectors and Associated Equipment (2014) http://dx.doi.org/10.1016/j.nima.2014.11.087.

19. M. Flaska, S.A. Pozzi, Identification of shielded neutron sources with the liquid scintillator BC-501A using a digital pulse shape discrimination method, Nuclear 
Instruments and Methods in Physics Research Section A: Accelerators, Spectrometers, Detectors and Associated Equipment 577 (3) (2007) 654-663.

20. J.L. Dolan, M. Flaska, A. Poitrasson-Riviere, A. Enqvist, P. Peerani, D.L. Chichester, S.A. Pozzi, Plutonium measurements with a fast-neutron multiplicity counter for nuclear safeguards applications,", Nuclear Instruments and Methods in Physics Research Section A: Accelerators, Spectrometers, Detectors and Associated Equipment 763 (2014) 565574.

21. G. Hull, N.P. Zaitseva, N.J. Cherepy, J.R. Newby, W. Stoeffl, S.A. Payne, New organic crystals for pulse shape discrimination, IEEE Transactions on Nuclear Science 56 (3) (2009) 899-903.

22. Yu.A. Kaschuck, B. Esposito, L.A. Trykov, V.P. Semenov, Fast neutron spectrometry with organic scintillators applied to magnetic fusion experiments, Nuclear Instruments and Methods in Physics Research Section A: Accelerators, Spectrometers, Detectors and Associated Equipment 476 (1-2) (2002) 511-515.

23. J.H. Baker, N.Z. Galunov, O.A. Tarasenko, Neutron scintillation detectors for environmental, security and geological studies, Proceedings of the IEEE Nuclear Science Symposium and Medical Imaging Conference, Honolulu, HI, October 27-November 3, 2007.

24. B-Series Fast, Blue-Sensitive Silicon Photomultiplier Sensors Datasheet, November 2014. URL (http://sensl.com/downloads/ds/DS-MicroBseries.pdf), downloaded on February 8, 2015.

25. C-Series Low Noise, Fast, Blue-Sensitive Photomultipliers Datasheet, December 2014. URL (http://sensl.com/downloads/ds/DS-MicroCseries.pdf), downloaded on February 8, 2015.

26. W.C. Kaiser, Scintillation spectrometry the state of the art, Analytical Chemistry 38 (11) (1966) 27A-39A.

27. Proteus Inc., Chagrin Falls, OH 44022, USA.

28. DS3153 - 730 Digitizer Family, February 14, 2014. URL (http://www.caen.it/csite/CaenProd.jsp?showLicence=false \&parent=62\&idmod=865), downloaded on February 5, 2015.

29. M. Flaska, S.A. Pozzi, Identification of shielded neutron sources with the liquid scintillator BC-501A using a digital pulse shape discrimination method, Nuclear Instruments and Methods in Physics Research Section A: Accelerators, Spectrometers, Detectors and Associated Equipment 577 (3) (2007) 654-663.

30. D. Cester, M. Lunardon, G. Nebbia, L. Stevanato, G. Viesti, S. Petrucci, C. Tintori, Pulse shape discrimination with fast digitizers, Nuclear Instruments and Methods in Physics 
314

Research Section A: Accelerators, Spectrometers, Detectors and Associated Equipment 748 (2014) 33-38.

31. A. Tomanin, J. Paepen, P. Schillebeeckx, R. Wynants, R. Nolte, A. Lavietes, Characterization of a cubic EJ-309 liquid scintillator detector, Nuclear Instruments and Methods in Physics Research Section A: Accelerators, Spectrometers, Detectors and Associated Equipment 756 (2014) 45-54.

32. J.R.M. Annand, A fast module for pulse shape analysis, Nuclear Instruments and Methods in Physics Research Section A: Accelerators, Spectrometers, Detectors and Associated Equipment 262 (2-3) (1987) 371-377.

33. G.F. Knoll, Radiation Detection and Measurement, John Wiley \& Sons, 2010. p. 701.

34. Inrad Optics Inc., Northvale, NJ 07647, USA. 